\title{
Tendon Healing in Bone Tunnel after Human Anterior Cruciate Ligament Reconstruction: A Systematic Review of Histological Results
}

\author{
Hongbin Lu, MD ${ }^{1}$ Can Chen, MD ${ }^{1}$ Shanshan Xie, MD 1 Yifu Tang, MD ${ }^{1} \quad$ Jin Qu, MD ${ }^{1}$ \\ ${ }^{1}$ Department of Sports Medicine \& Research Center of Sports \\ Medicine, Key Laboratory of Organ Injury, Aging and Regenerative \\ Medicine of Hunan Province, Xiangya Hospital, Central South \\ University, Changsha, People's Republic of China \\ Address for correspondence Jin Qu, MD, Department of Sports \\ Medicine \& Research Center of Sports Medicine, Key Laboratory of \\ Organ Injury, Aging and Regenerative Medicine of Hunan Province, \\ Xiangya Hospital, Central South University, Changsha 410008, \\ People's Republic of China (e-mail: jinqu@outlook.com).
}

J Knee Surg 2019;32:454-462.

Anterior cruciate ligament (ACL) rupture is one of the most common injuries in sports medicine. ${ }^{1}$ ACL reconstructions are usually performed to restore the stability of the knee joint. $^{2,3}$ Hamstring tendons are commonly used in ACL reconstruction because of the low harvest site morbidity compared with the patellar tendon. ${ }^{4}$ Reconstruction with hamstring tendons requires osteointegration of the tendon graft after initial fixation. Appropriate graft-to-bone healing is essential for postoperative rehabilitation and resumption of athletic activities. ${ }^{5}$ Although ACL reconstruction is performed with predictably good results, over thousands of articles have been published to understand how tendon received

February 24, 2018

accepted after revision

April 8, 2018

published online

May 21, 2018
Copyright (c) 2019 by Thieme Medical Publishers, Inc., 333 Seventh Avenue, New York, NY 10001, USA. Tel: +1(212) 584-4662.
DOI https://doi.org/ 10.1055/s-0038-1653964. ISSN 1538-8506. 
grafts heal to bone and how to improve tendon-to-bone healing in the past decades. ${ }^{6-10}$

Conventional mechanical testing has been regarded as a gold standard for the evaluation of tendon graft healing in bone tunnel. ${ }^{11-13}$ However, it is impossible to obtain enough human specimens for tensile testing to explore the tendon-to-bone healing process after ACL reconstruction with hamstring. Radiological evaluations are usually employed to explore the tendon-to-bone healing process, while histological evaluations could provide a direct and microscopic understanding. Due to the invasive nature of the biopsy procedure, most studies concerning to tendon healing and incorporation into bone are mainly based on animal studies. ${ }^{6,10}$ The evidence considering tendon graft healing to bone in humans is limited in several case series or case reports, and therefore, it is difficult to understand the healing process. The objective of this study was to systematically review the histological results of tendon healing in bone tunnel after human ACL reconstruction.

\section{Methods}

\section{Search Strategy}

A systematic review of the literature was performed in accordance with the Preferred Reporting Items for Systematic Reviews and Meta-analyses statement. ${ }^{14}$ An electronic search was performed using the following databases PubMed (until January 31, 2018), EMBASE (until January 31, 2018), Scopus (until January 31, 2018), and Cochrane Central Register of Controlled Trials (CENTRAL) databases (until January 31,2018 ). The computerized search was performed using the following search terms "(ACL reconstruction AND histology AND human) OR (ACL reconstruction AND histology AND human)."

\section{Study Selection}

Eligibility criteria: (1) ACL reconstruction with hamstring autograft, (2) tendon-to-bone interface tissue biopsy, and (3) tendon healing in bone tunnel was evaluated by histology. Exclusion criteria: (1) ACL reconstruction with bone-patellar tendon-bone graft, allografts, or artificial grafts and (2) only intra-articular graft was biopsied and evaluated.

\section{Study Quality Assessment}

The methodological quality of the selected studies was assessed with regard to study design, sample size, surgical techniques, detailed description of the biopsy procedure, use of independent or blinded examiners, and the adequate reporting of the techniques used to examine the specimens.

\section{Data Extraction}

Two reviewers independently extracted data from all eligible studies with a predeveloped data extraction form. The following information was collected: design of study, sample size, surgical techniques, graft type, graft fixation, resurgery indications for biopsy, biopsy procedures, techniques used to examine the samples, interval between ACL reconstruction and biopsy, and tendon-to-bone healing process. Any discrepancies between reviewers were resolved by consensus, and if necessary, a third reviewer would be consulted to make the final decision.

\section{Results}

\section{Literature Search}

The literature search totally generated 2,628 relevant citations from the four databases (-Fig. 1). After excluding the duplicates, there were 1,846 articles left. Subsequent review of the title/abstracts generated 11 articles that

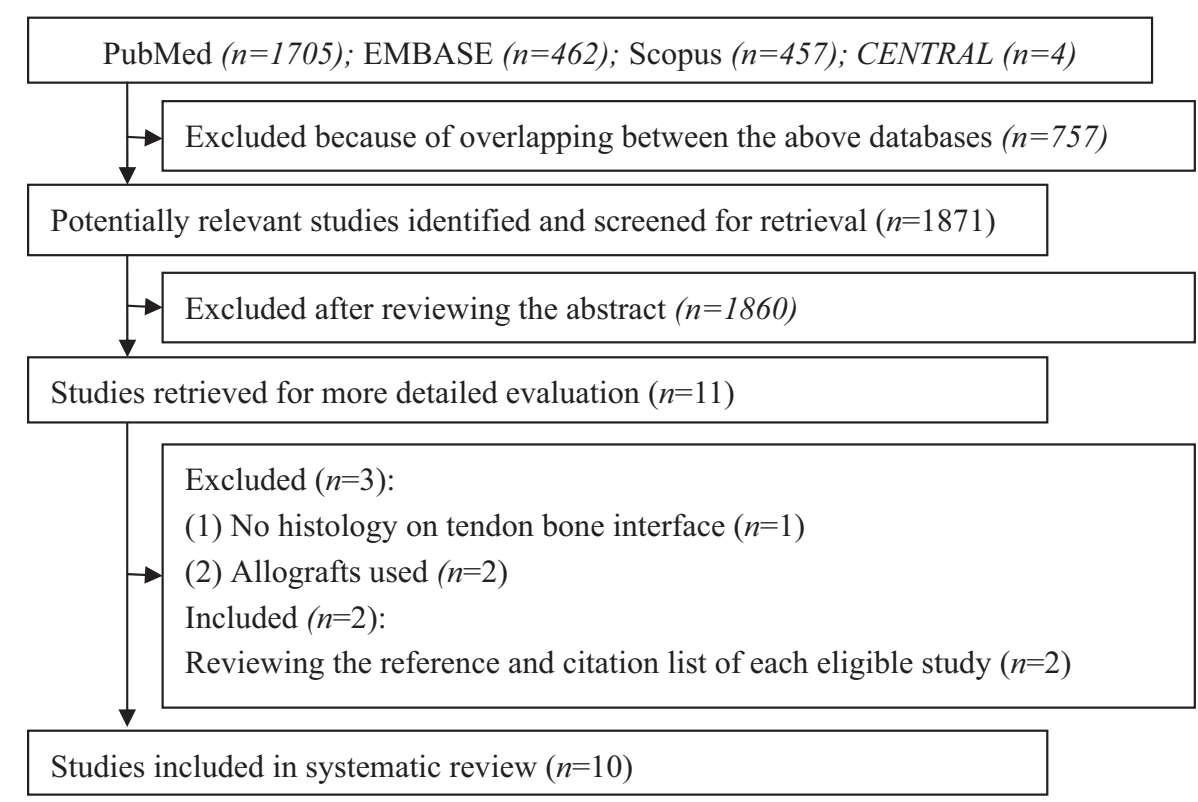

Fig. 1 The flow diagram illustrating the search process. 
were retrieved for further evaluation. ${ }^{15-25}$ One study was excluded because of no histology on tendon-bone interface ${ }^{25}$ Two articles were excluded due to allografts used. ${ }^{19,24}$ Two additional studies were included after reviewing the reference list of each eligible study. ${ }^{26,27}$ Finally, 10 studies were included in this systematic review. ${ }^{15-18,20-23,26,27}$

\section{Study Characteristics}

Five studies were conducted in European countries, ${ }^{17,18,20,21,23}$ two in North American countries, ${ }^{16,27}$ two in Australia, ${ }^{15,22}$ and one in Korea. ${ }^{26}$ Thirty-seven cases were extracted from the included studies. Seven of 10 studies reported the age of the patients, with a range of 14 to 42 years old. ${ }^{17,20-23,26,27}$ The interval between ACL reconstruction and biopsy ranged from 6 weeks to 3 years. The surgical techniques were variable among the included studies. Arthroscopic-assisted ACL reconstructions were only reported in four studies. ${ }^{15,21,22,27}$ The other six studies did not detail the surgical techniques. The graft fixation techniques greatly differed between reports. The main study characteristics are summarized in - Table $\mathbf{1}$.

\section{Study Quality}

Assessment of the methodologic quality of these studies revealed that no prospective studies could be retrieved owing to the invasive nature of the biopsy procedure. Four reports were case series (level of evidence 4). ${ }^{18,20,21,23}$ The remaining six reports were case reports (level of evidence $5) \cdot{ }^{15-17,22,26,27}$ Only one of the included reports used a blinded, independent examiner to evaluate the specimens. ${ }^{18}$

\section{Biopsy Procedure}

Most specimens were harvested during revision ACL reconstruction associated with traumatic graft failure or recurrent knee dysfunction (-Table $\mathbf{1}$ ). ${ }^{15-18,20-23,26}$ Ten specimens were obtained during TransFix device removal because of persistent pain on the lateral side of the knee. ${ }^{18}$ One specimen was obtained during knee resection for tibial osteosarcoma. ${ }^{27}$

The graft-bone interface tissues were mainly biopsied by coring a tunnel with a diameter of 4 to $10 \mathrm{~mm}$ and varied length ( - Table 1 ). Six specimens were obtained under arthroscopy with a diameter of 4 to $7 \mathrm{~mm}$ by a sharp spoon. ${ }^{18}$ Ten specimens were taken by coring a tunnel of $5 \mathrm{~mm}$ in diameter and $20 \mathrm{~mm}$ long along the femoral TransFix axis to obtain a cylindrical core of tissue composed of bone and ligament, $2 \mathrm{~cm}$ from the femoral outlet. ${ }^{21}$

\section{Study Techniques}

All samples were subjected to light microscopic evaluation. Sample preparation was similar in these reports with regard to fixation, dehydrating, and embedding of the specimens. Most specimens were stained using standard hematoxylin and eosin and Masson trichrome (-Table 2). ${ }^{15-17,21,23,27}$ Additionally, polarized light microscopy was used to explore the continuity of the collagen fibers of the tendon and bone in most studies (- Table 2). ${ }^{15,17,18,20-23,27}$ In all included studies, histologic changes during the graft-bone healing process were only qualitatively analyzed. None of the studies analyzed the histologic results in a quantitative way.

\section{Tendon Graft Healing in Bone Tunnel}

The study by Robert et al included 12 cases, with the largest sample size, and summarized the changes occurring in the healing of graft to bone and divided into three stages. ${ }^{21}$ None of the other studies analyzed the histological data based on different stages. The histological results of these studies were summarized based on the three stages ( $\mathbf{-}$ Table $\mathbf{2}$ ).

At 3 months, a fibrovascular interface was seen between the tendon and uncalcified osteoid with very few collagen fibers. One study reported that Sharpey-like fibers was visible at tibial tunnel as early as 6 weeks in one case. ${ }^{16}$ Another study also showed collagen fiber continuity between tibial bone tunnel and tendon at 3 to 4 months in two cases. ${ }^{15}$ The study by Lazarides et al included a case with knee resection at 4 months and revealed that collagen fibers were tightly interfaced into the surrounding bone with abundant bony ingrowth within the tibial tunnel, while there was an abundance of fat and no clear osseous infiltration within the femoral tunnel. There was abundant new bone formation and evidence of remodeling near the tibial screw insertion site. ${ }^{27}$

At 6 months, the fibrous interface was in continuous contact with the osteoid tissue covering lamellar bone, and Sharpey-like fibers started to be visible. One study by Nebelung et al included one case after ACL reconstruction using interference screw and revealed a firm attachment of tendinous tissue to the bone tunnel of femur at 8 months. ${ }^{20}$

At 10 months, mature indirect anchorage, characterizing Sharpey-like fibers, was obtained. The study by Heyde et al included five cases after ACL reconstruction with Synthes Milagro screw and revealed collagenous Sharpey-like fibers which extended from the tendon tissue radiated into the surrounding bone, with chondral metaplasia in the tibial tunnel at 8 to 24 months. ${ }^{23}$ The study by Petersen and Laprell included six cases after ACL reconstruction using EndoButton and Cramp and confirmed the healing of hamstring grafts in both tibial and femoral tunnels by formation of a fibrous insertion during 6 to 33 months. ${ }^{18}$ One study by Fankhauser et al included one case and revealed collagen-fibrous tendinous tissue with some myxoid degeneration, focal sclerosis, and scanty mesenchymal proliferation at the tibial and femoral articular tunnel aperture site at 2 years. The Sharpey-like collagenous fibrils could be detected by polarized light microscopy within the bone tunnel, along with atrophied tendinous tissue, fatty atrophic deposits, and focal regenerative vascular proliferation. ${ }^{22}$ The study by Eriksson et al showed that completed osseointegration of the graft had occurred with continuity of the collagen fibers of the tendon and tibial bone and formation of an interface resembling normal tendon to bone insertion in one case at 1 year. ${ }^{17}$

Overall, 10 cases from four studies were reported with negative results. The study by Nebelung et al included four cases after ACL reconstruction using the EndoButton or TransFix device and revealed granulation tissue between 


\begin{tabular}{|c|c|c|c|c|c|c|c|c|c|c|}
\hline 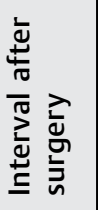 & 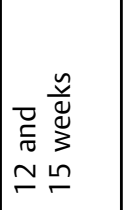 & \begin{tabular}{|l} 
产 \\
$\grave{3}$ \\
6
\end{tabular} & 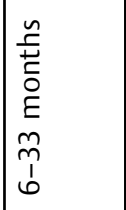 & 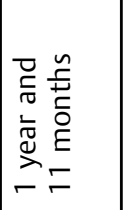 & 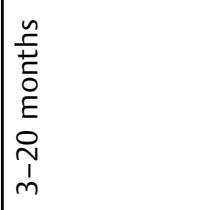 & 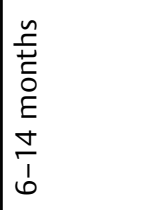 & 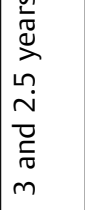 & 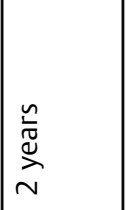 & 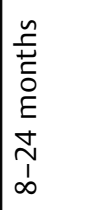 & 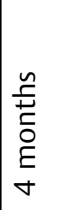 \\
\hline 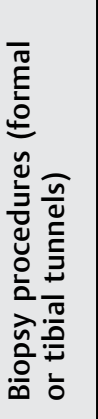 & 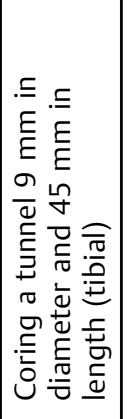 & 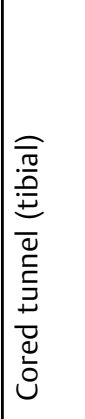 & 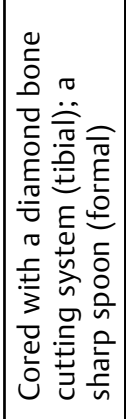 & 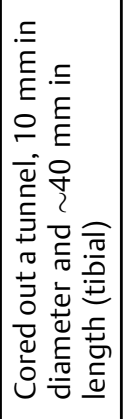 & 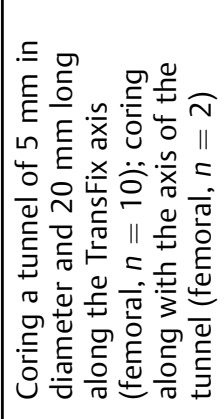 & 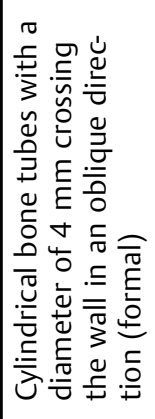 & 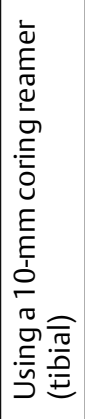 & 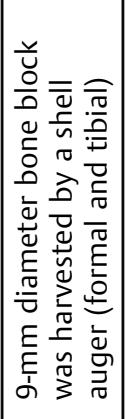 & 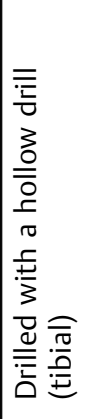 & 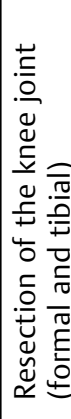 \\
\hline 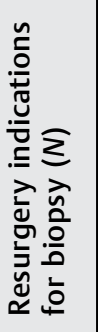 & 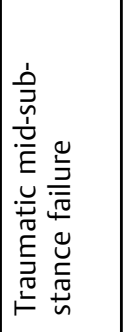 & 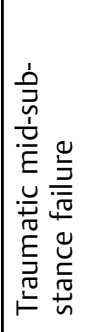 & 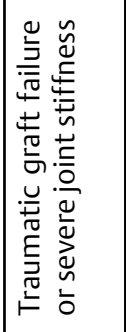 & 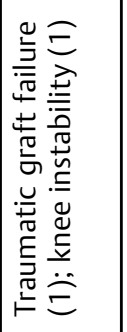 & 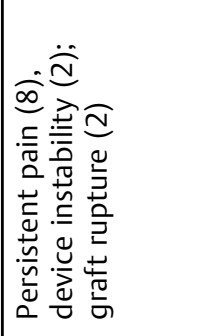 & 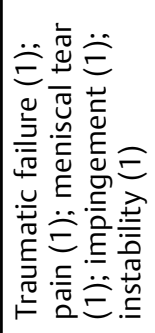 & 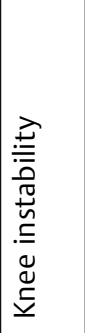 & 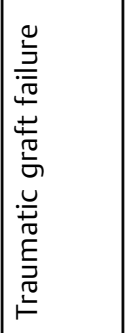 & 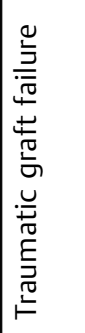 & 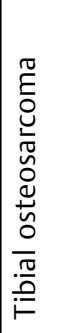 \\
\hline 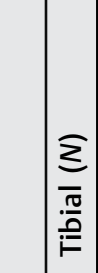 & 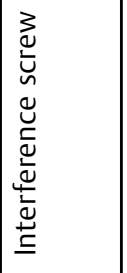 & 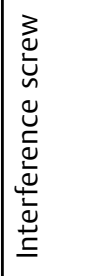 & \begin{tabular}{|l} 
产 \\
芯
\end{tabular} & 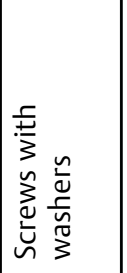 & 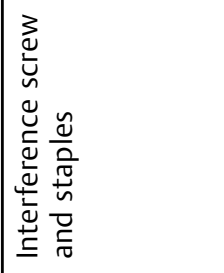 & 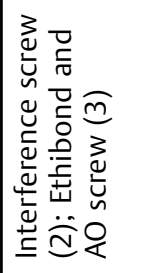 & $\begin{array}{l}\frac{\tilde{u}}{\frac{a}{a}} \\
\frac{\vec{N}}{\tilde{n}} \\
\sim\end{array}$ & 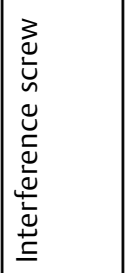 & 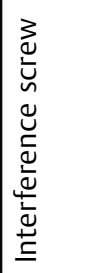 & 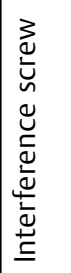 \\
\hline 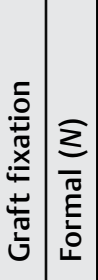 & 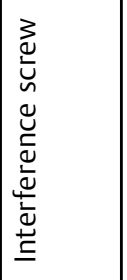 & 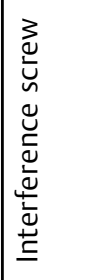 & 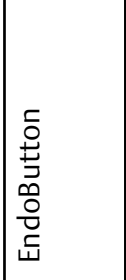 & 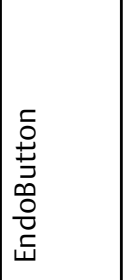 & 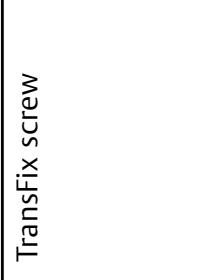 & 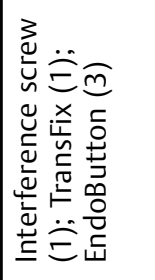 & 音 & 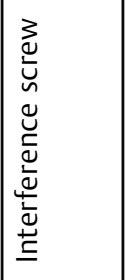 & 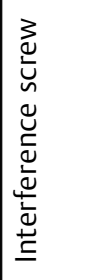 & 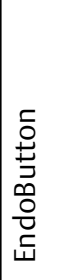 \\
\hline $\begin{array}{l}\frac{0}{a} \\
\text { Ev } \\
\stackrel{N}{n}\end{array}$ & $\sim$ & - & 6 & $\sim$ & 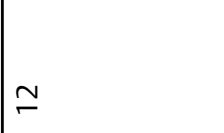 & in & $\sim$ & - & in & - \\
\hline 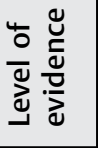 & in & in & F & in & $\nabla$ & $\nabla$ & เn & in & $\nabla$ & in \\
\hline & 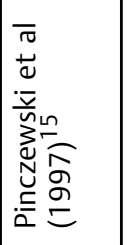 & 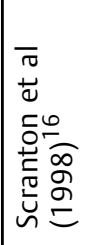 & 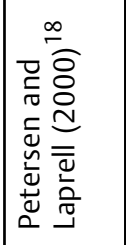 & 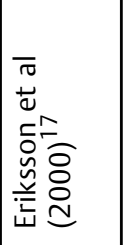 & 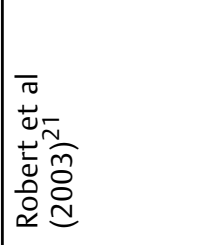 & 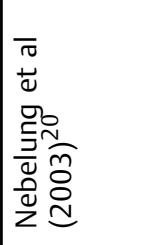 & 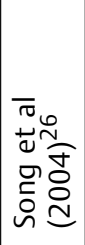 & 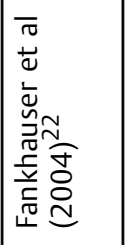 & 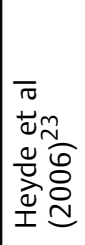 & 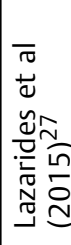 \\
\hline
\end{tabular}




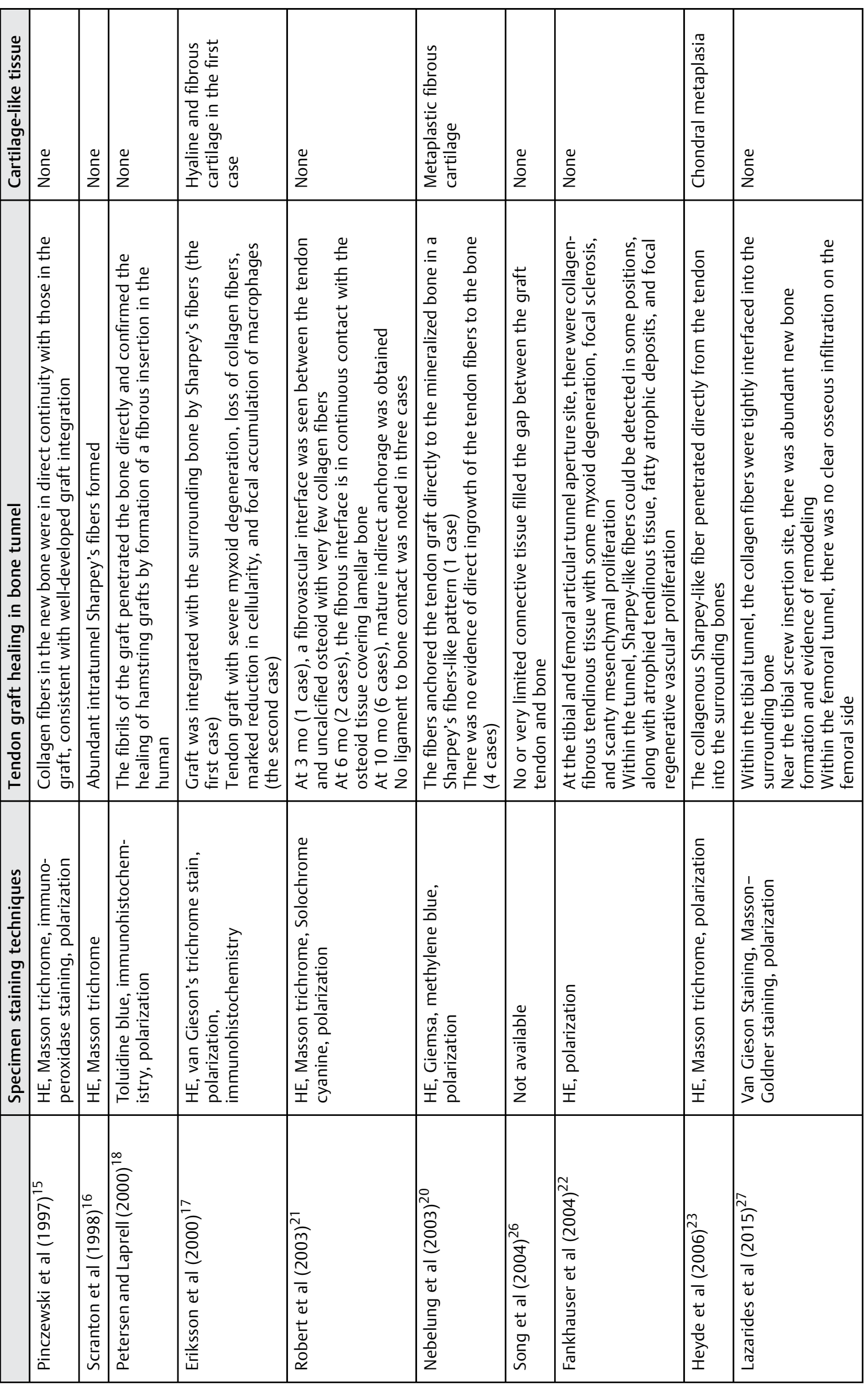


the graft and the bone without evidence of connecting collagen fibers at the femoral tendon-bone junction during 6 to 14 months. ${ }^{20}$ Eriksson et al included one case and reported that the tendon graft in tibial tunnel showed severe degenerative changes, the collagen fibers in many parts being dissolved and replaced by loose myxoid tissue, and areas of resorption with clusters of macrophages at 11 months. ${ }^{17}$ The study by Song et al included two cases with recurrent knee stability after ACL reconstruction with two staples, and showed no evidence of osteointegration or biologic remodeling of the tendon. ${ }^{26}$ Additionally, the study by Robert et al reported three cases in which no ligament to bone contact was noted despite good clinical stability at 12 to 15 months. ${ }^{21}$ Unexpectedly, suspensory type of fixation, including staples, screws with washers, TransFix and EndoButton, was used for the above 10 cases in which tendon graft failed to integrate with the surrounding bone.

Necrotic tendon graft was only seen in two cases from two studies at 6 to 12 weeks. ${ }^{15,16}$ Three studies explored the tendon-to-bone healing differences at different site $22,23,27$ and two studies revealed that tendon graft healed better at the articular tunnel aperture site than the intratunnel part. ${ }^{22,23}$ Cartilage-like tissue at tendon graft-bone interface was only reported in three studies in which interference screw was used for graft fixation ( - Table 2$)^{17,20,23}$

\section{Discussion}

Tendon graft healing in bone tunnel after ACL reconstruction has been explored widely in animal studies. Knowledge about tendon graft-bone healing process in human subjects is very limited due to the invasive and unethical nature. This study for the first time pooled all available studies to understand the healing process of tendon to bone after hamstring ACL reconstruction. The findings of the current systematic review indicated that a fibrovascular interface was formed between tendon graft and bone tunnel at early stage of 3 months. A fibrous interface in continuous contact with the bone tunnel could be expected at around 6 months. An indirect insertion, characterizing Sharpey-like fibers, tended to be matured after 10 months.

The femoral and tibial ACL insertions belong to fibrocartilaginous insertion and comprise four distinct zones: ligament, unmineralized fibrocartilage, mineralized fibrocartilage, and bone. ${ }^{28}$ This complex composite insertion is responsible for effectively transmitting the forces from ligament to bone. ${ }^{29,30}$ However, this study revealed that the fibrocartilaginous insertion was not regenerated after ACL reconstruction and a fibrous insertion, characterizing Sharpey-like fibers, was formed at tendon-bone interface instead. The outcomes following ACL reconstruction were reported to be generally good, with the success rates vary between 73 and $95 \%$, in the literature. ${ }^{31-33}$ The study by Robert et al indicated that tendon-to-bone healing was not always necessary for clinical stability of the knee joint. ${ }^{21}$ Rodeo et al studied the extensor tendon healing in the transtibial tunnel of dogs. At 12 and 24 weeks, polarized light microscopy showed evidence of collagen fibers connecting the tendon to the surrounding bone and tensile testing revealed that all specimens failed by pull-out of the tendon from the clamp or by mid-substance rupture of the tendon. Although a fibrocartilaginous insertion was not formed at the tendon-bone interface, tendon failed at the tendon-clamp junction or mid-substance of the tendon, not at the tendon-bone interface. ${ }^{34}$ These might explain why most patients are satisfied with the outcomes although a fibrocartilaginous insertion is not regenerated after ACL reconstruction.

A secure mechanical fixation is required to meet the demand of a rapid return of knee function in the early postoperative period before biologic incorporation of the graft in the bone tunnels. Many options are currently available that provide rigid fixation of hamstring graft. Commonly used fixation methods could be divided into two types: (1) fixation at the joint surface with interference screw and (2) fixation at a distance such as staples, screws with washers, TransFix and EndoButton. ${ }^{35}$ Weiler et al demonstrated that fixation at the joint surface using interference fit fixation might neutralize tendon graft-tunnel motions, and thus promoted the development of a direct type of ligament insertion at the joint surface. ${ }^{36}$ However, in this systematic review, cartilage-like tissue at tendon graft-bone interface was only reported in limited cases though interference screw was used for graft fixation. In the study by Weiler et al, the interference screw was inserted into the tibial tunnel in an inside-out direction, so that the head of the screw was flush with the intra-articular aperture of the tibial tunnel. ${ }^{36}$ Currently, the interference screw was usually inserted into the tibial tunnel in an outside-in direction, and this technique would reduce the compression of interference screw on the graft. Graft tunnel motion at the intra-articular aperture of the tibial tunnel would be expected in this technique, especially when the length of the interference screw is shorter than the length of the tibial tunnel. A direct fibrocartilaginous insertion was not regenerated in the included studies as that of Weiler et al's study, ${ }^{36}$ possibly because of graft motion existed at intra-articular aperture of bone tunnel. $^{6}$

The fixation of the hamstring tendon far from the tunnel aperture did not compress the tendon graft along the length of the tunnel as fixation with interference screw. This technique may allow the ingrowth of blood vessels along the entire length of the tendon graft. ${ }^{35}$ In this study, 37 cases were included for analysis. Unexpectedly, there were 10 cases failed to integrate tendon graft with the surrounding bone and suspensory type of fixation was used in these failure cases. Early intratunnel motion of the graft was considered to preclude graft tendon healing to bone. Graft motion would be expected when suspensory type of fixation was used. The study by Lazarides et al explored the tendonto-bone healing differences between interference screw versus EndoButton, and indicated that fixation with interference screw leading to an indirect ligamentous insertion and fixation with EndoButton leading to viability of the graft 
but with very little evidence of a healing response. ${ }^{27}$ Although fixation with EndoButton may lead to slow healing process, the success rate of ACL reconstruction with EndoButton is up to $95.8 \% .{ }^{37}$ A recent meta-analysis by Browning et al revealed that there was significantly better knee stability in the suspensory fixation group compared with the aperture fixation group and the graft rupture rate was lower with suspensory fixation compared with aperture fixation. ${ }^{38}$ The security of the graft is more reliant on tunnel wall integrity at aperture fixation than suspensory fixation. This might explain why there was better knee stability in the suspensory fixation group and reconfirmed that tendonto-bone healing was not always necessary for clinical stability of the knee joint.

It has been suggested that differences in the histologic appearance of the tendon-to-bone attachment on different sides of the bone tunnel. Weiler et al demonstrated that intratunnel healing was only important during early healing stages. When solid surface healing occurred, the intratunnel part of the graft tissue might be stress shielded, resulting in a partial resorption and continuous tunnel narrowing over time. ${ }^{36}$ This was confirmed by Fankhauser et al's study, in which Sharpey-like collagen fibers was found bridging the bone and the graft, along with atrophied tendinous tissue, fatty atrophic deposits, and focal regenerative vascular proliferation. ${ }^{22}$ The study by Lazarides et al revealed that the collagen fibers were tightly interfaced into the surrounding bone within bone tunnel and there was abundant new bone formation and evidence of remodeling at the aperture site. ${ }^{27}$ The study by Weiler et al found a direct ligament insertion formed at the joint surface with these specific transition zones after tendon graft implantation into a bone tunnel. ${ }^{36}$ Both studies by Fankhauser et al and Lazarides et al did not reveal a direct ligament insertion formed at the joint surface although interference screw was used for graft fixation. ${ }^{22,27}$ In contrast, Rodeo et al employed a rabbit ACL reconstruction model with suspensory fixation and demonstrated that tendon-to-bone healing was slower at the intra-articular tunnel aperture than at the tunnel exit. ${ }^{39}$ This difference might be owing to the presence of graft tunnel motion in the grafts fixed with suspensory fixation.

It is clear that tendon-to-bone healing process is a continuum of biologic changes rather than a series of distinct, time-dependent biologic events. Based on animal studies, tendon graft healing in bone tunnel could be divided into four stages: (1) inflammatory phase, (2) proliferative phase, (3) matrix synthesis, and (4) matrix remodeling. ${ }^{7,10}$ An infiltration and recruitment of inflammatory cells and stem cells could result in the release of cytokines and growth factors, leading to an ingrowth of blood vessels and nerves. In the current review, a fibrovascular interface was developed at 3 months, probably corresponding to the inflammatory phase and proliferative phase. During the matrix synthesis phase, collagen fibers form at tendon-bone interface. At 6 months, the fibrous interface was in continuous contact with the surrounding bone, probably corresponding to matrix synthesis phase. At the matrix remodeling phase, the newly formed graft-bone interface remodeled with mature collagen fibers formed between tendon graft and bone tunnel. At 10 months, mature indirect insertion with Sharpey-like fibers was formed, probably corresponding to matrix remodeling phase. However, due to the lack of enough high-quality studies, it is far from understanding of the tendon-to-bone healing process.

There are several limitations existed in this review. First, all the included studies were case series or case reports. There were massive differences in surgical techniques, graft fixations, and rehabilitation protocols among included studies. The level of evidence of these reports was too poor to draw a definite conclusion. Further studies with standard surgical and fixation techniques are highly needed to understand the healing process of tendon to bone. Second, due to the invasive nature of the biopsy procedure and its potential deleterious effects, most specimens included were harvested during revision ACL reconstruction associated with traumatic graft failure or recurrent knee dysfunction. Only one study retrieved an entire knee joint from a boy with osteosarcoma of the tibia. The graft healing process in this case was similar to that reported in several included studies. The biopsy location of the tunnel was not clear among included studies. Standardized biopsy procedures would help provide a better understanding of tendon graft healing to bone in human ACL reconstruction. Third, most included studies only employed standard staining methods, not immunohistochemical methods. Certain specific proteins or cell types of the healing interface could not be explored. The histological results of all eligible studies were only qualitatively analyzed. Future studies with large sample size may include immunohistochemistry and qualitative analysis. Fourth, 10 of 37 (27\%) cases were reported with negative results in the systematic review. Most specimens were harvested during revision ACL reconstruction. The rate of negative results might have been overestimated. In addition, the graftbone interface was unloaded during the time of trauma and revision surgery. This might also affect the healing process of tendon graft in bone tunnel. These results might not be entirely representative.

In summary, the key finding of this systematic review is that an indirect type of insertion with Sharpey-like fibers between tendon and bone would be expected after ACL reconstruction with hamstring. Cartilage-like tissue may be formed in the tendon-bone interface occasionally. A firm tendon-to-bone healing was not always necessary for clinical stability of the knee joint. The underlying graft bone healing process is far from understood in the human ACL reconstruction with hamstring. Further human studies are highly needed to understand tendon graft healing in bone tunnel after hamstring ACL reconstruction.

\section{Funding}

This study was funded by the National Natural Science Foundation of China (81501898) and Central South University (2016zzts124). 


\section{Conflict of Interest}

None declared.

\section{References}

1 Griffin LY, Agel J, Albohm MJ, et al. Noncontact anterior cruciate ligament injuries: risk factors and prevention strategies. J Am Acad Orthop Surg 2000;8(03):141-150

$2 \mathrm{HuJ}$, Qu J, Xu D, Zhou J, Lu H. Allograft versus autograft for anterior cruciate ligament reconstruction: an up-to-date meta-analysis of prospective studies. Int Orthop 2013;37(02):311-320

$3 \mathrm{Hu} \mathrm{J}, \mathrm{Qu} \mathrm{J}, \mathrm{Xu} \mathrm{D}$, Zhang T, Zhou J, Lu H. Clinical outcomes of remnant preserving augmentation in anterior cruciate ligament reconstruction: a systematic review. Knee Surg Sports Traumatol Arthrosc 2014;22(09):1976-1985

4 Chechik O, Amar E, Khashan M, Lador R, Eyal G, Gold A. An international survey on anterior cruciate ligament reconstruction practices. Int Orthop 2013;37(02):201-206

5 Magnussen RA, Trojani C, Granan LP, et al; MARS Group; SFA Revision ACL Group. Patient demographics and surgical characteristics in ACL revision: a comparison of French, Norwegian, and North American cohorts. Knee Surg Sports Traumatol Arthrosc 2015;23(08):2339-2348

6 Ekdahl M, Wang JH, Ronga M, Fu FH. Graft healing in anterior cruciate ligament reconstruction. Knee Surg Sports Traumatol Arthrosc 2008;16(10):935-947

7 Lui P, Zhang P, Chan K, Qin L. Biology and augmentation of tendonbone insertion repair. J Orthop Surg 2010;5:59

8 Atesok K, Fu FH, Wolf MR, et al. Augmentation of tendon-to-bone healing. J Bone Joint Surg Am 2014;96(06):513-521

9 Kuang GM, Yau WP, Lu WW, Chiu KY. Osteointegration of soft tissue grafts within the bone tunnels in anterior cruciate ligament reconstruction can be enhanced. Knee Surg Sports Traumatol Arthrosc 2010;18(08):1038-1051

10 Herbst E, Albers M, Kopka M, Shaikh H, Fu FH. Biology of graft incorporation. Asian J Arthrosc 2016;1(01):20-24

$11 \mathrm{Hu} \mathrm{J}, \mathrm{Qu} \mathrm{J}, \mathrm{Xu} \mathrm{D}$, Zhang T, Qin L, Lu H. Combined application of lowintensity pulsed ultrasound and functional electrical stimulation accelerates bone-tendon junction healing in a rabbit model. J Orthop Res 2014;32(02):204-209

12 Xu D, Zhang T, Qu J, Hu J, Lu H. Enhanced patella-patellar tendon healing using combined magnetic fields in a rabbit model. Am J Sports Med 2014;42(10):2495-2501

$13 \mathrm{Lu} \mathrm{H}$, Chen C, Qu J, et al. Initiation timing of low-intensity pulsed ultrasound stimulation for tendon-bone healing in a rabbit model. Am J Sports Med 2016;44(10):2706-2715

14 Moher D, Liberati A, Tetzlaff J, Altman DG; PRISMA Group. Preferred reporting items for systematic reviews and meta-analyses: the PRISMA statement. BMJ 2009;339:b2535

15 Pinczewski LA, Clingeleffer AJ, Otto DD, Bonar SF, Corry IS. Integration of hamstring tendon graft with bone in reconstruction of the anterior cruciate ligament. Arthroscopy 1997;13(05): 641-643

16 Scranton PE Jr, Lanzer WL, Ferguson MS, Kirkman TR, Pflaster DS. Mechanisms of anterior cruciate ligament neovascularization and ligamentization. Arthroscopy 1998;14(07):702-716

17 Eriksson K, Kindblom LG, Wredmark T. Semitendinosus tendon graft ingrowth in tibial tunnel following ACL reconstruction: a histological study of 2 patients with different types of early graft failure. Acta Orthop Scand 2000;71(03):275-279

18 Petersen W, Laprell H. Insertion of autologous tendon grafts to the bone: a histological and immunohistochemical study of hamstring and patellar tendon grafts. Knee Surg Sports Traumatol Arthrosc 2000;8(01):26-31

19 Yamakado K, Kitaoka K, Nakamura T, et al. Histologic analysis of the tibial bone tunnel after anterior cruciate ligament reconstruc- tion using solvent-dried and gamma-irradiated fascia lata allograft. Arthroscopy 2001;17(08):32

20 Nebelung W, Becker R, Urbach D, Röpke M, Roessner A. Histological findings of tendon-bone healing following anterior cruciate ligament reconstruction with hamstring grafts. Arch Orthop Trauma Surg 2003;123(04):158-163

21 Robert H, Es-Sayeh J, Heymann D, Passuti N, Eloit S, Vaneenoge E. Hamstring insertion site healing after anterior cruciate ligament reconstruction in patients with symptomatic hardware or repeat rupture: a histologic study in 12 patients. Arthroscopy 2003;19 (09):948-954

22 Fankhauser F, Passler JM, Schippinger G, Boldin C, Scarpatetti M. Tendon-to-bone healing of a quadrupled hamstring tendon graft fixed with biodegradable screws in an immature athlete: a radiologic, arthroscopic, histologic, and electromicroscopic investigation. Arthroscopy 2004;20(09):992-997

23 Heyde CE, Wiechmann V, Pyschik M, Gosse A. Histological findings for human tibial bone-tendon healing after hamstring transplantation. Investigation of tibial insertion after reconstruction of anterior cruciate ligaments with hamstring transplantation near joint fixation using bioresorbable interference screws [in German]. Unfallchirurg 2006;109(05):377-382

24 Lee CA, Meyer JV, Shilt JS, Poehling GG. Allograft maturation in anterior cruciate ligament reconstruction. Arthroscopy 2004;20 (Suppl 2):46-49

25 Logan M, Williams A, Myers P. Is bone tunnel osseointegration in hamstring tendon autograft anterior cruciate ligament reconstruction important? Arthroscopy 2003;19(08):E1-E3

26 Song EK, Rowe SM, Chung JY, Moon ES, Lee KB. Failure of osteointegration of hamstring tendon autograft after anterior cruciate ligament reconstruction. Arthroscopy 2004;20(04): 424-428

27 Lazarides AL, Eward WC, Green K, Cardona DM, Brigman BE, Taylor DC. Histological evaluation of tendon-bone healing of an anterior cruciate ligament hamstring graft in a 14-year-old boy. Am J Sports Med 2015;43(08):1935-1940

28 Evans EJ, Benjamin M, Pemberton DJ. Fibrocartilage in the attachment zones of the quadriceps tendon and patellar ligament of man. J Anat 1990;171:155-162

29 Genin GM, Thomopoulos S. The tendon-to-bone attachment: unification through disarray. Nat Mater 2017;16(06):607-608

30 Rossetti L, Kuntz LA, Kunold E, et al. The microstructure and micromechanics of the tendon-bone insertion. Nat Mater 2017; 16(06):664-670

31 Fithian DC, Paxton EW, Stone ML, et al. Prospective trial of a treatment algorithm for the management of the anterior cruciate ligament-injured knee. Am J Sports Med 2005;33(03): 335-346

32 Yunes M, Richmond JC, Engels EA, Pinczewski LA. Patellar versus hamstring tendons in anterior cruciate ligament reconstruction: a meta-analysis. Arthroscopy 2001;17(03):248-257

33 Freedman KB, D’Amato MJ, Nedeff DD, Kaz A, Bach BR Jr. Arthroscopic anterior cruciate ligament reconstruction: a metaanalysis comparing patellar tendon and hamstring tendon autografts. Am J Sports Med 2003;31(01):2-11

34 Rodeo SA, Arnoczky SP, Torzilli PA, Hidaka C, Warren RF. Tendon-healing in a bone tunnel. A biomechanical and histological study in the dog. J Bone Joint Surg Am 1993;75(12): 1795-1803

35 Robbe R, Paletta GA Jr. Soft-tissue graft fixation in anterior cruciate ligament reconstruction. Oper Tech Sports Med 2004; 12(03):188-194

36 Weiler A, Hoffmann RF, Bail HJ, Rehm O, Südkamp NP. Tendon healing in a bone tunnel. Part II: histologic analysis after biodegradable interference fit fixation in a model of anterior cruciate ligament reconstruction in sheep. Arthroscopy 2002;18(02): 124-135 
462 Tendon Healing in Bone Tunnel Lu et al.

37 Tian S, Wang Y, Wang B, et al. Anatomic double-bundle anterior cruciate ligament reconstruction with a hamstring tendon autograft and fresh-frozen allograft: a prospective, randomized, and controlled study. Arthroscopy 2016;32(12):2521-2531

38 Browning WM III, Kluczynski MA, Curatolo C, Marzo JM. Suspensory versus aperture fixation of a quadrupled hamstring tendon autograft in anterior cruciate ligament reconstruction: a metaanalysis. Am J Sports Med 2017;45(10):2418-2427

39 Rodeo SA, Kawamura S, Kim HJ, Dynybil C, Ying L. Tendon healing in a bone tunnel differs at the tunnel entrance versus the tunnel exit: an effect of graft-tunnel motion? Am J Sports Med 2006;34 (11):1790-1800 